J. Clin. Chem. Clin. Biochem.

Vol. 19, 1981, pp. 1125-1130

\title{
Quantitative lasernephelometrische Bestimmung von Rheumafaktoren mittels IgG-beschichteter Latexpartikel
}

\author{
Von K. Husmann, H. J. Lakomek, O. Richter, E. Jacobi und H. L. Krüskemper \\ Medizinische Klinik und Poliklinik C (Direktor: Prof. Dr. med. H. L. Krüskemper) der Universität Düsseldorf
}

(Eingegangen am 26. November 1980/1. Juni 1981)

Zusammenfassung: Der lasernephelometrische Latex-Rheumafaktor-Test (Latex-RF-Test) ist geeignet, durch Immunkomplexbildung zwischen IgG-beschichteten Latexpartikeln und Rheumafaktoren Rheumafaktor-Aktivität nachzuweisen. Die Methode erfordert geringen zeitlichen Aufwand und liefert mit guter Präzision quantitative Werte. Beim qualitativen Nachweis sind die Ergebnisse des lasernephelometrischen Testes und der am häufigsten angewandten Agglutinationsreaktionen (Waaler-Rose- und Latex-Agglutinationstest) gleich. Beim Vergleich der Ergebnisse der quantitativen Messungen mit den entsprechenden Waaler-Rose- und Latex-Agglutinationstesttitern sind höheren Titern im Mittel höhere lasernephelometrische Werte zugeordnet. Die hohe Streuung der Einzelwerte legt jedoch nahe, daß weitere Faktoren neben den IgM-Rheumafaktoren, deren Konzentration im wesentlichen die Höhe der Titer in den Agglutinationsreaktionen bestimmt, das Ausmaß der Immunkomplexbildung beeinflussen (IgG-Rheumafaktoren, IgG- und IgG-IgM-Immunkomplexe).

\section{Quantitative laser-nephelometric detection of rheumatoid factor, using IgG coated latex particles}

Summary: The laser-nephelometric test (Latex RF test) detects rheumatoid factor activity, and is based on immune complex formation between IgG coated latex particles and rheumatoid factor. This test requires little time and gives quantitative results with good precision. The latex nephelometric test and the frequently used agglutination tests (Waaler-Rose, Latex agglutination test) give the same qualitative results. In the quantitative assay higher nephelometric results correlate on average with higher titers in the Waaler-Rose and Latex agglutination test. The high standard deviation could be explained by the fact that other factors (IgG rheumatoid factor, IgG and IgG-IgM immune complexes), in addition to the IgM rheumatoid factor concentration, influence the immune complex formation in vitro.

\section{Ëinleitung}

Bei etwa achtzig Prozent aller Patienten mit einer adulten rheumatoiden Arthritis lassen sich Rheumafaktoren (Antigàmmaglobulinfaktoren) im Serum nachweisen. Es handelt sich um spezifische, gegen Fc-Fragment von IgG-Immunglobulin gerichtete polyklonale Antikörper, vorwiegend der IgM-, aber auch der IgG- und IgA-Immunglobulinklasse. Obwohl sie auch bei den anderen sogenannten Kollagenosen und einigen weiteren chronisch entzündlichen Erkrankungen auftreten, wird Ihnen eine diagnostische Wertigkeit zuerkannt. Der Rheumafaktor-Test ist eines der diagnostischen Kriterien der Arthritis and Rheumatism Association von 1958 (1) und 1963 (2) und auch in den New Yorker Kriterien furr rheumatoide Arthritis vôn 1966 enthalten.

Der Nachweis sollte quantitativ geführt werden, da Rheumafaktoren niedrigtitrig unspezifisch häufiger auch bei Gesunden, besonders im höheren Lebensalter, nachgewiesen werden $(3,4)$. Bei höheren Titern nimmt die
Spezifität im Mittel zu, auch bestehen Hinweise, daß zwischen der Höhe des Rheumafaktor-Titers und dem klinischen Schweregrad und der Prognose eine Beziehung besteht $(3,5,6)$. Die semiquantitative Bestimmung der Rheumafaktor-Aktivität erfolgt üblicherweise als Endpunkttitration mit dem Waaler-Rose-oder Latex-Agglutinationstest nach Singer \& Plotz (7-9). Der quantitative Nachweis ist mittels radioimmunologischer (10-14), fluoreszenzoptischer (15-17) oder enzymimmunologischer $(18,19)$ Techniken möglich, die in der Regel sehr aufwendig und eher wissenschaftlichen Fragestellungen vorbehalten sind. Lasernephelometrische Verfahren sind weniger aufwendig und liefern ebenfalls quantitative Ergebnisse $(9,15,20,21)$. Durch Präzipitation hitzeaggregierten IgG's oder IgG-beschichteter Latexpartikel wird eine meßbare Trübung hervorgerufen. Das Ausmaß der Trübung ist proportional der Antigammaglobulin-Konzentration $(20,21)$. Im Folgenden werden die Ergebnisse eines neuen lasernephelometrischen Testes mit 
IgG-beschichteten Latexpartikeln als Indikator beschrieben. Die Entwicklung des Latex-Reagenzes erfolgte durch die Behring Lab. Marburg (Vertrieb in Vorbereitung). Der Test erscheint aufgrund seiner Praktikabilität besonders für die routinemäßige quantitative Rheumafaktor-Bestimmung geeignet. Die Ergebnisse werden zu denen des Waaler-Rose- und Latex-Agglutinationstestes in Bezug gesetzt.

\section{Methodik}

Alle untersuchten Seren sind Nüchternseren und werden bis zur Untersuchung bei $-20^{\circ} \mathrm{C}$ gelagert.

Alle lasernephelometrischen Messungen erfolgen mit einem Behring Laser-Nephelometer (He-Ne-Laser 632,8 nm, Meßbereich $0-20 \mathrm{~V}$ ) und speziellen Plastikküvetten (Behring, Marburg).

Die Bestimmung der Rheumafaktor-Aktivität durch Stufentitration erfolgt mittels kommerzieller Tests: Rheumaton ${ }^{\circledR}$ (BykMallinckrodt, modifiziert nach Waaler-Rose) und Latex-Agglutinationstest ${ }^{\circledR}$ (Behring, nach Singer \& Plotz). Alle Seren werden nach dem Auftauen durch Erhitzen komplementinaktiviert $\left(30 \mathrm{~min}\right.$ bei $\left.56^{\circ} \mathrm{C}\right)$. Eventuelle Trübungen werden durch Ausschütteln mit Frigen ${ }^{\circledR}$ (Behring, Marburg) beseitigt. Zwei Teile Frigen ${ }^{\circledR}$ werden mit einem Teil Serum gemischt (Vortex) und abzentrifugiert, der Überstand enthält das geklärte Serum.

Die Kalibrierung erfolgt mit einem anhand original WHO-Rheumafaktor-Standard kalibrierten Rheumafaktor-Referenz-Serum (Behring, Marburg). Die lyophilisierte Trockensubstanz wird mit destill. Wasser gelöst und enthält $156 \times 10^{-3} \mathrm{IE} / \mathrm{l}$. Nach Millipore-Filtration $(0,45 \mu \mathrm{m})$ und Hitzeinaktivierung wird mit Glycin-Albumin-Pufferlösung eine geometrische Verdünnungsreihe mit den Stufen 1:65, 1:130, 1:260, 1:520 und 1:1040 angelegt, die Referenzkurve reicht von $0,15 \times 10^{-3}$ bis $2,4 \times$ $10^{-3} \mathrm{IE} / \mathrm{l}$.

Die Verdünnung der Seren erfolgt, wenn nicht anders beschrieben, 1:100 mit Glycin-Albumin-Pufferlösung, die ebenfalls Millipore-filtriert wird $(0.45 \mu \mathrm{m})$. Der anhand der Referenzkurve beurteilbare Meßbereich reicht also von $15 \times 10^{-3}$ bis $240 \times$ $10^{-3} \mathrm{IE} / \mathrm{l}$. Ist der Meßwert der Lichtstreuungsintensität gleich dem Meßwert der 1:65-Verdünnung des Standards (entsprechend $\left.240 \times 10^{-3} \mathrm{IE} / \mathrm{l}\right)$, wird die Messung mit einer 1:800-Verdünnung wiederholt (Meßbereich: $120 \times 10^{-3}$ bis $1920 \times 10^{-3} \mathrm{IE} / \mathrm{l}$ ). $150 \mu \mathrm{l}$ der jeweiligen Serumverdünnungen (des Standards) werden mit $75 \mu$ l Latex-Rheumafaktor-Reagenz (Behring, Marburg) in der Meßküvette gemischt (Vortex) und, wenn nicht anders beschrieben, eine Stunde bei Raumtemperatur inkubiert. Die eintretende Trübung wird im Laser-Nephelometer gemessen.

\section{Ergebnisse}

\section{Reaktionskinetik}

Bei unterschiedlich langer Inkubation von Rheumafaktor-Aktivität enthaltendem Serum mit Latex-Rheumafaktor-Reagenz kommt es zunächst zu einem zeitabhängigem Anstieg der Streulichtintensität. Die Steilheit des Anstieges ist größer bei Seren, die im Waaler-Rose- und Latex-Agglutinationstest noch bei höheren Verdünnungen positiv reagieren. Nach etwa drei- bis fünfstïndiger Inkubation wird bei mittel- und hochtitrigen Seren die maximale Streulichtintensität gemessen. Bei längerer Inkubationsdauer sinkt die Streulichtintensität ab. Ursache für das Absinken der Streulichtintensität ist möglicherweise die Sedimentation größerer Immunkomplexaggregate. Denkbar ist auch eine Dissoziation der Komplexe, allerdings steigen Seren, in denen Rheumafaktor-
Aktivität mittels der Agglutinations-Reaktion lediglich bei niedrigen Verdünnungsstufen nachweisbar ist, bei Inkubation über 24 Stunden kontinuierlich an. Abbildung 1 zeigt einige typische Kurvenverläufe. Die verschiedenen Standardverdünnungen zeigen ein jeweils der enthaltenden Rheumafaktor-Aktivität entsprechendes Verhalten. Rheumafaktor-negative Seren (Waaler-Rose-, Latex-Agglutinationstest) zeigen keinen Anstieg (Abb. 1).

Meßwerte bei unterschiedlichen Serumverdünnungen

Die meßbare Rheumafaktor-Konzentration wird beim überwiegenden Teil der Seren durch unterschiedliche Vorverdünnungen nicht oder nur gering beeinflußt. Von 21 Seren mit niedrigem und mittelhohem.Rheumafaktor-Titer im Waaler-Rose- und Latex-Agglutinationstest wird eine lineare Verdünnungsreihe mit Glycin-AlbuminPufferlösung von $1: 25$ bis $1: 1600$, von weiteren $21 \mathrm{Se}$ ren mit mittlerem und hohem Rheumafaktor-Titer von $1: 100$ bis $1: 25600$ angelegt. Die anhand der Referenzkurve aus dem Streulichtsignal unter Berücksichtigung der Verdünnungsstufe ermittelten Rheumafaktor-Konzentrationen der Seren weichen jeweils nur wenig voneinander ab (Abb. 2a), dàs Streulichtsignal ist der größeren Verdünnung entsprechend niedriger. Sehr geringe und sehr hohe Serumverdünnungen bedingen ein Meßsignal, das außerhalb des Meßbereiches der Referenzkurve liegt.

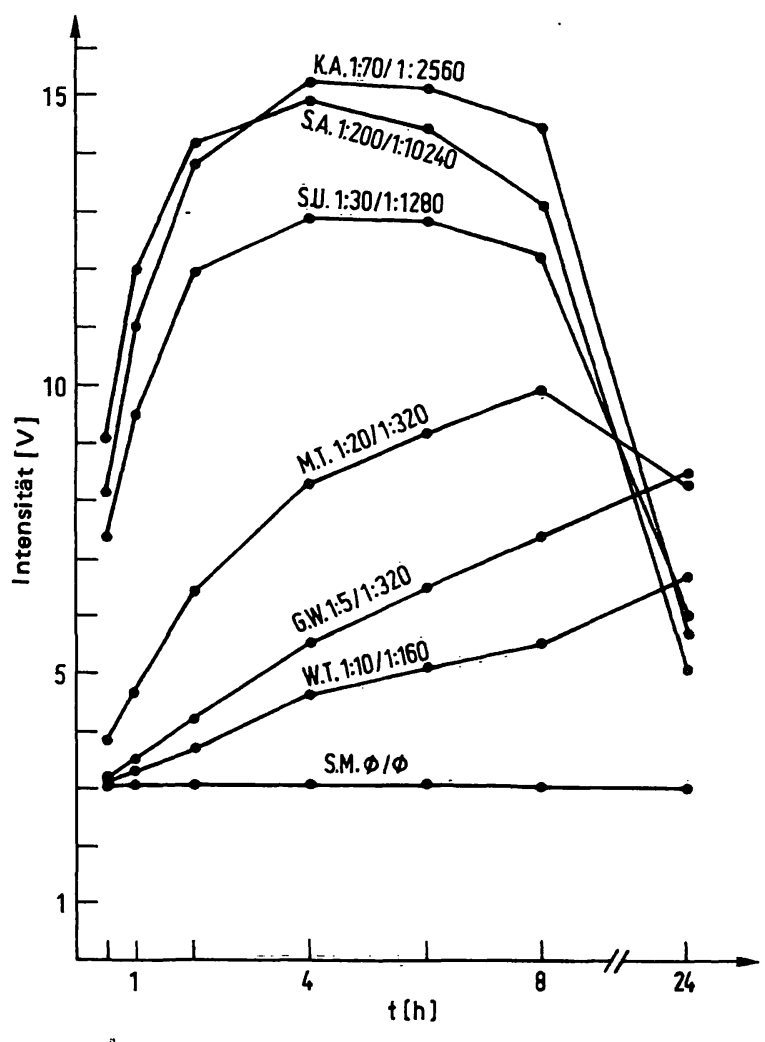

Abb. 1. Abhängigkeit des Streulichtsignals von der Inkubationszeit des Reaktionsansatzes bei sechs Seren mit verschieden hohen Rheumafaktor-Titern im Waaler-Rose- und Latex-Agglutinationstest und einem iheumafaktor-negativen Serum. 

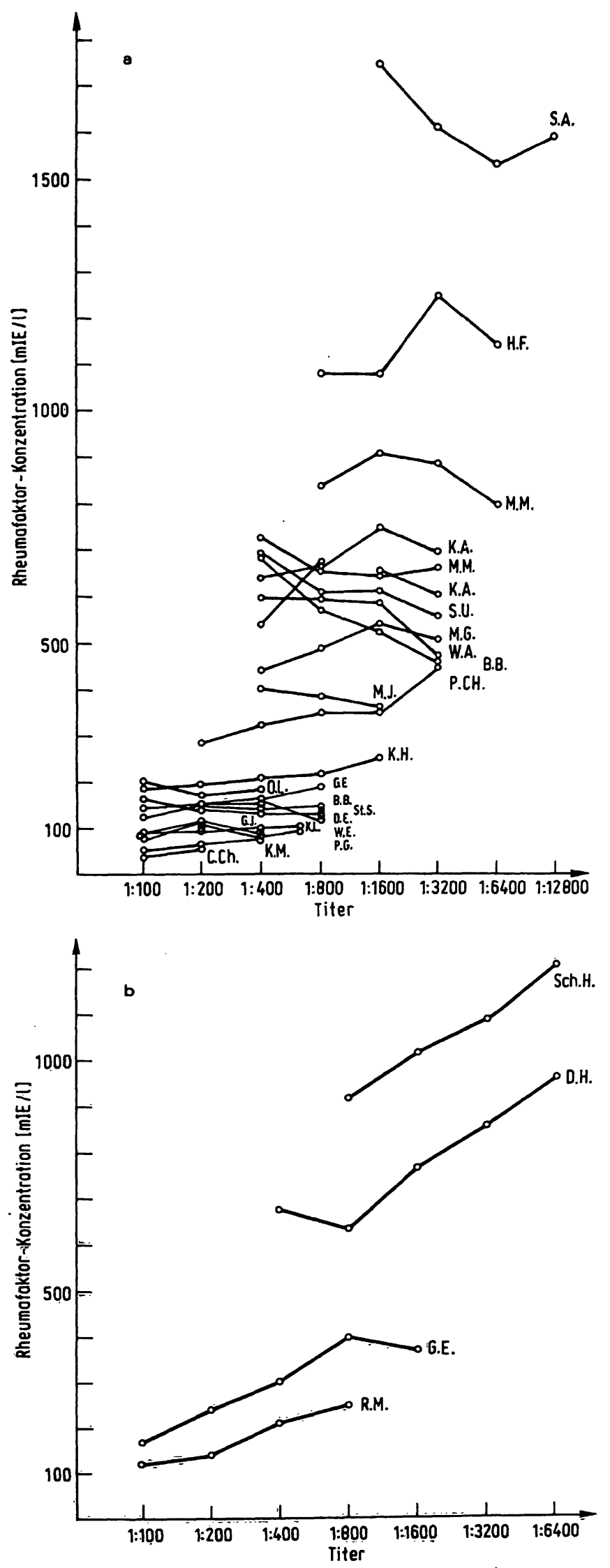

Abb. 2. Rheumafaktor-Konzentrationen bei unterschiedlicher Vorverdünnung des Ausgangsserums (errechnet aus der Höhe des Streulichtsignals anhand der Referenzkurve unter Berücksichtigung des Verdünnungsfaktors).

a: Weitgehend konstante Rheumafaktor-Konzentration

b: Relativ ansteigende Rheumafaktor-Konzentration bei zunehmend höherer Verdünnung.
Bei einigen Seren sinkt das Streulichtsignal zwar ebenfalls mit zunehmender Serumvorverdünnung, jedoch nicht entsprechend der Verdünnungsstufe, so daß die anhand der Referenzkurve ermittelten Rheumafaktor-Konzentrationen unter Berücksichtigung der Verdünnungsstufe ansteigen (Abb. 2b).

Bei weiteren 28 Seren mit hoher Rheumafaktor-Aktivität (Waaler-Rose-, Latex-Agglutinationstest) findet sich bei niedriger Serumverdünnung $(1: 25,1: 50)$ Streulichtintensität im Meßbereich der Referenzkurve, bei mittlerer Verdünnungsstufe ( $1: 100$ bis 1:800) wird ein Streulichtsignal oberhalb des Meßbereiches gemessen. Bei hoher Serumvorverdünnung sinkt die Streulichtintensität in den Meßbereich zurück.

\section{Präzision}

Die Präzision der gemessenen Rheumafaktor-Konzentrationen in der Serie wird durch Mehrfachbestimmungen $(n=10)$ anhand von vier Seren geprüft. Die Aliquots werden bis zum Tag der Messung bei $-20^{\circ} \mathrm{C}$ gelagert. Die Ergebnisse (Mittelwert, Meßbereich, Standardabweichung und Variationskoeffizient) zeigt Tabelle 1.

Tab. 1. Präzision des Latex-RF-Testes bei Tag-zu-Tag-Messung.

\begin{tabular}{|c|c|c|c|c|c|}
\hline \multirow{2}{*}{$\begin{array}{l}\text { Se- } \\
\text { rum }\end{array}$} & \multirow{2}{*}{$\begin{array}{l}\text { Titer } \\
\text { Waaler-Rosel } \\
\text { Latex }\end{array}$} & \multicolumn{4}{|c|}{ Rheumafaktoraktivität $\left(10^{-3} \mathrm{IE} / 1\right)$ Varia- } \\
\hline & & $\begin{array}{l}\text { Mittel- } \\
\text { wert } \\
(n=10)\end{array}$ & Meßbereich & $\begin{array}{l}\text { Standard- } \\
\text { abwei- } \\
\text { chung }\end{array}$ & $\begin{array}{l}\text { tions- } \\
\text { koeffi- } \\
\text { zient } \\
(\%)\end{array}$ \\
\hline $\begin{array}{l}1 \\
2 \\
3 \\
4\end{array}$ & $\begin{array}{l}1: 5 / 1: 320 \\
1: 20 / 1: 320 \\
1: 40 / 1: 1280 \\
1: 150 / 1: 5120\end{array}$ & $\begin{array}{l}28,85 \\
39,1 \\
402 \\
856\end{array}$ & $\begin{array}{r}22-46 \\
34-44 \\
360-440 \\
800-1000\end{array}$ & $\begin{array}{r}7,45 \\
3,21 \\
25,66 \\
63,1\end{array}$ & $\begin{array}{r}25,4 \\
8,2 \\
6,4 \\
7,4\end{array}$ \\
\hline
\end{tabular}

\section{Sensitivität}

\section{Empfindlichkeitsgrenze des Latex-RF-Testes}

Die untere Empfindlichkeitsgrenze des Latex-RF-Testes beträgt bei anhand der Referenzkurve ermittelten quantitativen Werten $15 \times 10^{-3} \mathrm{IE} / \mathrm{l}$. Während der kumulative Leerwert von Küvette und Serumverdünnung zwischen 0,05 und $0,1 \mathrm{~V}$ beträgt und vernachlässigt werden kann, liegt der Leerwert des Latex-Rheumafaktor-Reagenzes im Mittel bei 2,51 V ( $\mathrm{n}=72,2,30-2,85 \mathrm{~V}, \mathrm{~s}=0,11 \mathrm{~V}$, $3 s=0,33 \mathrm{~V})$. Der Meßwert der 1:1040-Verdünnung des Standards (entsprechend $15 \times 10^{-3} \mathrm{IE} / \mathrm{l}$ ) beträgt im Mittel $3,28 \mathrm{~V}(\mathrm{n}=72,2,89-3,58 \mathrm{~V}, \mathrm{~s}=0,15 \mathrm{~V}, 3 \mathrm{~s}=$ $0,45 \mathrm{~V})$. Seren, deren Meßwert geringer als der der $1: 1040$-Standardverdünnung mit $15 \times 10^{-3} \mathrm{IE} / \mathrm{l}$ ist, können nicht sicher vom Leerwert unterschieden werden.

\section{Empfindlichkeitssteigerung durch 24stündige Inkubation}

In Seren mit einer Rheumafaktor-Konzentration von weniger als $15 \times 10^{-3} \mathrm{IE} / 1$ kann der Nachweis von Rheu- 
mafaktor-Aktivität qualitativ durch Inkubation des Reaktionsansatzes über 24 Stunden geführt werden. Das Meßsignal steigt deutlich über den Meßwert des Leerwertes an. Der Leerwert und Seren von Gesunden, in denen auch mit dem Waaler-Rose- und Latex-Agglutinationstest keine Rheumafaktor-Aktivität nachweisbar ist, weisen keinen Anstieg auf.

\section{Empfindlichkeit des Latex-RF-Testes im Vergleich zum Waaler-Rose- und Latex-Agglutinationstest}

Die Nachweisgrenze der Agglutinationsreaktionen wird mit dem anhand eines WHO-Standards kalibrierten Rheumafaktor-Standard (Behring) bestimmt (156 X

$10^{-3} \mathrm{IE} / \mathrm{l}$ ). Eine deutliche Agglutination ist beim modifizierten Waaler-Rose-Test (Rheumaton ${ }^{\circledR}$ ) noch bei einer Verdünnung auf $5 \times 10^{-3} \mathrm{IE} / 1$ zu erzielen, beim Latex-Agglutinationstest auf $4 \times 10^{-3} \mathrm{IE} / \mathrm{l}$. Entsprechend der Referenzkurve liegt bei quantitativer Messung die untere Meßbereichsgrenze des lasernephelometrischen Latex-RF-Testes bei $15 \times 10^{-3} \mathrm{IE} / \mathrm{l}$.

In Seren mit geringerer Rheumafaktor-Konzentration läßt sich lasernephelometrisch der Nachweis qualitativ führen (Inkubation über 24 Stunden). Von 350 Seren mit einem Rheumafaktortiter zwischen 1:1 und 1:200 im Waaler-Rose-Test lassen sich lasernephelometrisch bei 264 Seren Rheumafaktor-Konzentrationen zwischen $15 \times 10^{-3}$ und $3840 \times 10^{-3} \mathrm{IE} / 1$ messen; ein qualitativer Rheumafaktor-Nachweis gelingt bei weiteren 73 , von diesen weisen 62 einen Waaler-Rose-Titer von 1:5 und weniger auf. Bei 13 Seren (mit einem Waaler-Rose-Titer von 1:10 und weniger) lassen sich lasernephelometrisch keine Rheumafaktoren nachweisen. Diese Seren stammen von Patienten mit juveniler rheumatoider Arthritis $(n=2)$, systemischem Lupus erythematodes $(n=2)$, Morbus Behcet $(n=1)$, Psoriasis-Arthritis $(n=1)$ und nicht dem rheumatologischen Formenkreis zugehörigen verschiedenen Erkrankungen ( $n=7)$. Ähnliche Ergebnisse zeigt der Vergleich mit dem Latex-Agglutinationstest: Bei 326 Seren mit einem Rheumafaktor-Titer zwischen 1:20 und $=1: 40960$ finden sich lasernephelometrisch Rheumafaktor-Konzentrationen zwischen $15 \times 10^{-3}$ und $3840 \times$ $10^{-3} \mathrm{IE} / 1$ bei 260 , bei weiteren 56 ( 51 mit Titern von 1:160 und weniger) Konzentrationen von weniger als $15 \times 10^{-3} \mathrm{IE} / 1$. Bei 10 Seren mit niedrigen Latex-Titern gelingt lasernephelometrisch der Rheumafaktor-Nachweis nicht, 8 Seren stammen von Patienten ohne Erkrankungen aus dem rheumatologischen Formenkreis (Tab. 2a, b). Bei 128 Seren ohne Nachweis von Rheumafaktor-Aktivität mit den Agglutinationstesten fällt der lasernephelometrische Test $11 \mathrm{mal}$ positiv aus, die Konzentrationen liegen immer unter $15 \times 10^{-3} \mathrm{IE} / 1$.

Rheumafaktor-Nachweis bei Patienten mit rheumatoider Arthritis

Von 79 Patienten mit einer rheumatoiden Arthritis lassen sich bei 62 Rheumafaktoren mit dem Waaler-Roseund Latex-Agglutinationstest nachweisen, bei 2 Patienten
Tab. 2. Empfindlichkeit des Latex-RF-Testes im Vergleich zum Waaler-Rose- und Latex-Agglutinationstest.

a: Häufigkeit des lasernephelometrischen RheumafaktorNachweises (Latex-RF-Test) bei Seren, in denen Rheumafaktor-Aktivität mit dem Waaler-Rose-Test bei niedriger $(\leqslant 1: 2)$, mittlerer $(1: 5)$ und hoher Verdünnungsstufe $(\geqslant 1: 10)$ nachweisbar ist. Bei Seren mit positivem lasernephelometrischen Rheumafaktor-Test wird die Anzahl der Seren mit höherer Konzentration $\left(>15 \times 10^{-3} \mathrm{IE} / \mathrm{l}\right)$ und niedriger Konzentration $\left(<15 \times 10^{-3} \mathrm{IE} / \mathrm{l}\right.$, mit positivem Reaktionsanfall erst nach vierundzwanzigstündiger Inkubation) angegeben.

\section{Waaler-Rose-Test Latex-RF-Test}

\begin{tabular}{|c|c|c|c|}
\hline $\begin{array}{l}\text { positiv } \\
n=350\end{array}$ & $\begin{array}{l}\text { positiv } \\
n=337\end{array}$ & & $\begin{array}{l}\text { negativ } \\
n=13\end{array}$ \\
\hline Titer. & $>15 \times 10^{-3} \mathrm{IE} / 1$ & $<15 \times 10^{-3} \mathrm{IE} / \mathrm{l}$ & \\
\hline $\begin{array}{l}\leqslant 1: 2 \\
n=46\end{array}$ & 10 & 30 & 6. \\
\hline $\begin{array}{l}1: 5 \\
n=67\end{array}$ & 31 & 32 & 4 \\
\hline $\begin{array}{l}1: 10 \\
n=237\end{array}$ & 223 & 11 & 3 \\
\hline
\end{tabular}

$\mathrm{b}$ : Entsprechende Zahlen der Seren, in denen RheumafaktorAktivität bei niedriger $(\leqslant 1: 160)$, mittlerer $(1: 320)$ und hoher $(\geqslant 1: 640)$ Verdünnung mittels des Latex-Agglutinationstestes nachweisbar ist.

\begin{tabular}{lccl}
\hline $\begin{array}{l}\text { Latex- } \\
\text { Agglutinationstest }\end{array}$ & Latex-RF-Test & & \\
$\begin{array}{l}\text { positiv } \\
n=326\end{array}$ & $\begin{array}{l}\text { positiv } \\
n=316\end{array}$ & negativ \\
Titer & $>15 \times 10^{-3} \mathrm{IE} / 1$ & $<15 \times 10^{-3} \mathrm{IE} / \mathrm{l}$ & \\
\hline $\begin{array}{l}\mathrm{k} 1: 160 \\
\mathrm{n}=101\end{array}$ & 43 & 51 & 7 \\
$\begin{array}{l}1: 320 \\
\mathrm{n}=52\end{array}$ & 47 & 3 & 2 \\
$\begin{array}{l}1: 640 \\
\mathrm{n}=173\end{array}$ & 170 & 2 & 1 \\
\hline
\end{tabular}

gelingt der Nachweis niedrigtitrig nur mit jeweils einer der Agglutinationsreaktionen. Bei diesen 64 Patienten (81\%) finden sich lasernephelometrisch ebenfalls Rheumafaktoren in unterschiedlichen Konzentrationen. Bei den übrigen 15 Patienten ist auch lasernephelometrisch ein Rheumafaktor-Nachweis nicht möglich.

Korrelation von Rheumafaktor-Konzentrationen (Laser) mit Titerhöhe (Waaler-Rose-/Latex-

Agglutinationstest)

Bei 230 Seren mit bekanntem Latex-Agglutinationstiter und 217 mit bekanntem Waaler-Rose-Titer werden lasernephelometrisch die Rheumafaktor-Konzentrationen bestimmt; die den jeweiligen Titerstufen des Waaler-Roseund Latex-Agglutinationstestes zuzuordnenden Meß̧werte zeigt Tabelle 3a, b. Die für jede Titerstufe errechneten Mittelwerte der Rheumafaktor-Konzentrationen steigen mit zunehmender Titerstufe bei allerdings weiter Uber- 

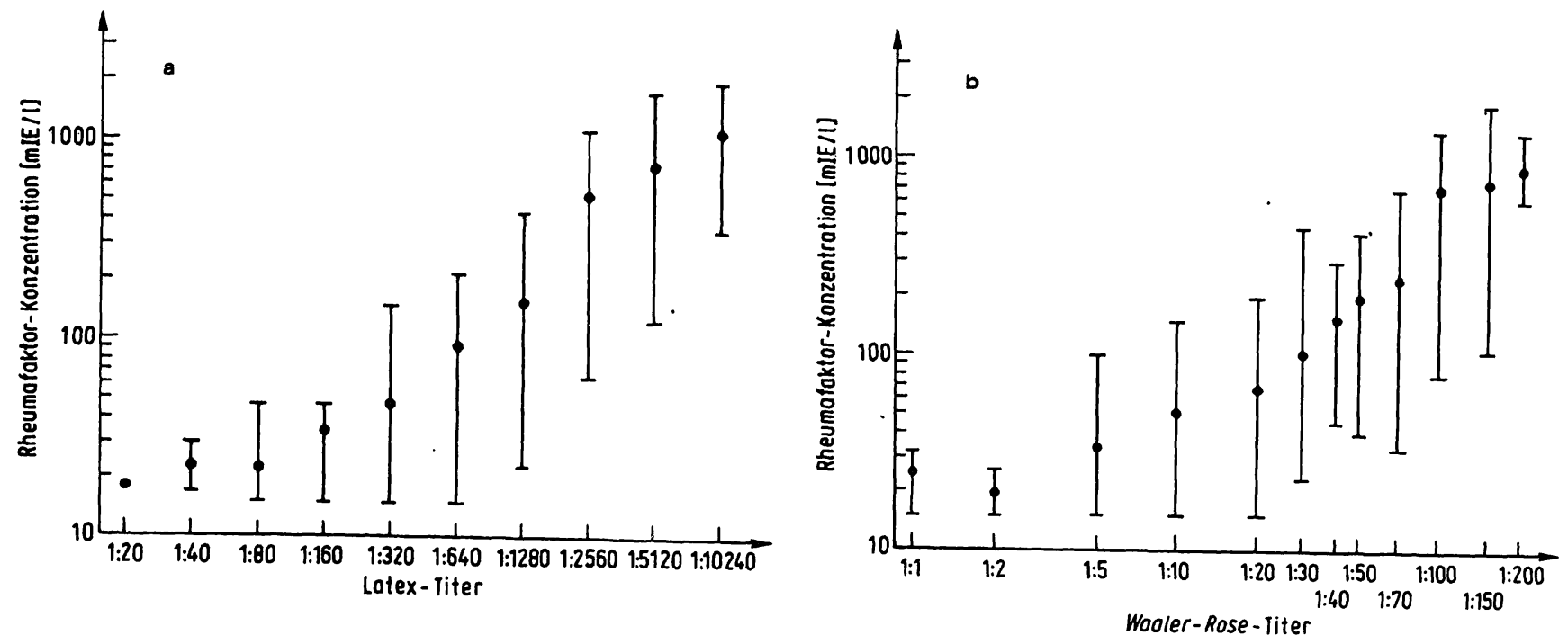

Abb. 3. Rheumafaktor-Konzentrationen (Latex-RF-Test) in Korrelation zu den Titerstufen der Agglutinationsreaktionen. Aufgetragen sind jeweils Mittelwert und Schwankungsbreite der Einzelmessungen, tabelliert dic Angabe der Standardabweichung und die Anzahl der in jeder Titerstufe zur Auswertung kommenden Seren.

a: lasernephelometrischer Latex-RF-Test/Latex-Agglutinationstest

b: lasernephelometrischer Latex-RF-Test/Waaler-Rose-Test

Abb. 3a

Wertepaare

\begin{tabular}{|c|c|c|c|c|c|c|c|c|c|c|}
\hline $\begin{array}{l}\text { Latex-Titer } \\
\text { c Rheumafaktor, } \bar{x}(\mathrm{mIE} / \mathrm{l}) \\
\text { s (mIE/l) } \\
\text { n }\end{array}$ & $\begin{array}{l}1: 20 \\
17 \\
- \\
1\end{array}$ & $\begin{array}{l}1: 40 \\
22 \\
6 \\
4\end{array}$ & $\begin{array}{l}1: 80 \\
23 \\
10 \\
11\end{array}$ & $\begin{array}{l}1: 160 \\
35 \\
21 \\
25\end{array}$ & $\begin{array}{l}1.320 \\
48 \\
30 \\
43\end{array}$ & $\begin{array}{l}1: 640 \\
91 \\
48 \\
33\end{array}$ & $\begin{array}{l}1: 1280 \\
163 \\
104 \\
43\end{array}$ & $\begin{array}{l}1: 2560 \\
530 \\
372 \\
28\end{array}$ & $\begin{array}{l}1: 5120 \\
878 \\
393 \\
25\end{array}$ & $\begin{array}{l}1: 10240 \\
1178 \\
554 \\
17\end{array}$ \\
\hline
\end{tabular}

Abb. 3b

Wertepaare

\begin{tabular}{|c|c|c|c|c|c|c|c|c|c|c|c|c|}
\hline $\begin{array}{l}\text { Waaler-Rose-Titer } \\
\text { c Rheumafaktor, } \bar{x}(\mathrm{mIE} / \mathrm{l}) \\
\text { s (mIE/l) } \\
\text { n }\end{array}$ & $\begin{array}{l}1: 1 \\
25 \\
8 \\
5\end{array}$ & $\begin{array}{c}1: 2 \\
19 \\
4 \\
8\end{array}$ & $\begin{array}{l}1: 5 \\
34 \\
21 \\
29\end{array}$ & $\begin{array}{l}1: 10 \\
52 \\
34 \\
42\end{array}$ & $\begin{array}{l}1: 20 \\
70 \\
49 \\
26\end{array}$ & $\begin{array}{l}1: 30 \\
104 \\
103 \\
18\end{array}$ & $\begin{array}{l}1: 40 \\
165 \\
79 \\
16\end{array}$ & $\begin{array}{c}1: 50 \\
192 \\
99 \\
20\end{array}$ & $\begin{array}{l}1: 70 \\
288 \\
259 \\
13\end{array}$ & $\begin{array}{l}1: 100 \\
735 \\
398 \\
13\end{array}$ & $\begin{array}{l}1: 150 \\
794 \\
445 \\
23\end{array}$ & $\begin{array}{l}1: 200 \\
899 \\
387 \\
3\end{array}$ \\
\hline
\end{tabular}

schneidung der Meßbereiche ebenfalls an, zwischen der gemessenen Rheumafaktor-Konzentration und der Höhe der Titerstufe besteht eine positive Korrelation, der Spearman'sche Rangkorrelationskoeffizient beträgt für die Beziehung Waaler-Rose-Titer und Latex-RF-Test 0,83 , für die Beziehung Latex-Agglutinationstest und Latex-RF-Test 0,87 .

\section{Disküssion}

Der Latex-RF-Test ist ein neuer, lasernephelometrischer Test zum qualitativen und gleichzeitig quantitativen Nachweis von Rheumafaktoren. Er ist gut praktikabel und erfordert nur geringen zeitlichen Aufwand. Die Meßergebnisse in der Tag-zu-Tag-Messung sind ausgezeichnet reproduzierbar, die Präzision ist hoch.

Die Empfindlichkeit entspricht der der herkömmlichen Agglutinationsreaktionen, die Nachweisbarkeit von Rheumafaktoraktivität in Seren von Patienten mit einer rheumatoiden Arthritis ist mit dem lasernephelometrischen Test identisch den Agglutinationsreaktionen.
Agglutinationsreaktionen und Latex-RF-Test scheinen dasselbe Substrat nachzuweisen: Im untersuchten Kollektiv rheumafaktor-positiver wie -negativer Seren (Waaler-Rose-/Latex-Agglutinationstest) von Patienten, die nicht nach der zugrunde liegenden Krankheit unterschieden werden (u.a. Rheumatoide Arthritis, andere Bindegewebserkrankungen, schwere chronische Allgemeinerkrankungen) und Gesunder finden sich lasernephelometrisch Rheumafaktoren in nahezu allen ,seropositiven" und nur vereinzelt in den „seronegativen" Seren.

Beim qualitativen Nachweis von Rheumafaktor-Aktivität zeigt der Latex-RF-Test den Agglutinationsreaktionen entsprechende Ergebnisse. Im Gegensatz zum WaalerRose- und Latex-Agglutinationstest, die mittels Endpunkttitration lediglich relativ ungenaue, semiquantitative Werte in Form von Titerstufen liefern, kann mit dem lasernephelometrischen Test die Rheumafaktor-Aktivität direkt quantitativ bestimmt werden. Während bei den Agglutinationsreaktionen als Maß die Titerstufe angegeben wird, bei der die Sekundärreaktion der AntigenAntikörper-Reaktion (Agglutination) gerade noch sicht- 
bar ist, wird beim lasernephelometrischen Latex-RF-Test die Immunkomplexbildung mittels der eintretenden Trübung direkt gemessen (22). Die gemessene Streulichtintensität hängt sowohl von der Immunkomplex-Konzentration wie auch von der Größe der Komplexe ab (23). Die nach Heidelberger (12) zu erwartende Abhängigkeit der Komplexkonzentration und damit des Streulichtes vom Antigen-Antikörper-Verhältnis scheint allerdings bei konstanter Antigenvorgabe (Latex-RF-Reagenz) nur gering oder nicht vorhanden zu sein.

Bei einem kleineren Teil der Seren findet sich hingegen bei weiterer Vorverdünnung des Serums ein deutlicher relativer Anstieg des Streulichtes und der unter Berücksichtigung des Verdünnungsfaktors errechneten Rheumafaktor-Konzentration. Die Ursache für dieses abweichende Verhalten ist unklar. Möglicherweise kommt es in diesen Seren bei zunehmender Verdünnung zur Dissoziation von Immunkomplexen aus dimeren IgG-Rheumafaktoroder IgG-IgM-Komplexen. Bei Durchführung des Testes als Routinemethode könnten diskrepante Rheumafaktor-Konzentrationen in zwei ausreichend verschiedenen Serumverdünnungen (z.B. 1:100 und 1:800) als Hinweis auf das Vorhandensein von Rheumafaktor-Immunkomplexen in vivo dienen. $\mathrm{Da}$ insbesondere IgG-Rheumafaktor- und IgG-Rheumafaktor-enthaltende Komplexe mit in die pathogenetischen Mechanismen der Erkrankung involviert sein sollen (6), ergibt sich hier möglicherweise eine einfache Möglichkeit zur qualitativen Erweiterung der Rheumadiagnostik. Die Ergebnisse bedürfen allerdings noch weiterer Uberprüfung.
Ein direkter Vergleich der durch Endpunkttitration beim Waaler-Rose- und Latex-Agglutinationstest bestimmten Rheumafaktor-Aktivität mit den quantitativen Meßwerten des lasernephelometrischen Latex-RF-Testes ist aus den vorgenannten Gründen problematisch. Seren mit noch in höheren Titerstufen positivem Rheumafaktornachweis sind zwar im Mittel lasernephelometriṣch höhere Rheumafaktor-Konzentrationen zugeordnet, es besteht eine positive Korrelation (Spearman'scher Rangkorrelationskoeffizient), die den jeweiligen Titerstufen zuzuordnenden Einzelwerte weisen allerdings eine erhebliche Streuung auf. Die benachbarten Titerstufen zugeordneten Mittelwerte der lasernephelometrischen Einzelmessungen sind nicht signifikant verschieden. Da mit der Agglutinationsreaktion in erster Linie IgM-Rheumafaktoren erfaßt werden, ist die Titerhöhe im wesentlichen durch die IgMRheumafaktor-Aktivität bedingt. Die beobachtete Streuung der Einzelwerte könnte durch eine relativ. größere Bedeutung von Rheumafaktoren anderer Immunglobulinklassen (IgG, IgA) und möglicherweise vorhandenen Immunkomplexen (IgG-Di- und Polymere, IgG-IgMKomplexe) für das Ausmaß der Immunkomplexbildung in vitro bedingt sein.

Zusammenfassend ist der lạsernephelometrische LatexRF-Test einfach, präzise und schnell in der Durchführung. Rheumafaktoren werden mit gleicher Häufigkeit nachgewiesen wie bei den Agglutinationsreaktionen, die quantitative Messung erlaubt Titerverlaufskontrollen mit geringem Aufwand. Möglicherweise ist der Test geeignet, eine Aussage zum eventuellen Vorhandensein von Immunkomplexen (mit Rheumafaktor-Beteiligung) zu mächen.

\section{Literatur}

1. Ropes, M. W., Bennet, E. A., Cobb, S., Jacox, R. \& Jessar, R. (1958) Bull. Rheum. Dis. 9, 175-176.

2. Blumberg, B. S., Bunim, Ch. J., Calkins, E., Pirani, C. L. \& Zvaifler, N. J. (1964) Arthritis Rheum. 1, 93-97.

3. Cats, A. \& Klein, F. (1970) Ann. Rheum. Dis. 29, 663-672.

4. Valkenburg, H. A., Ball, J., Burch, T. A., Bennett, P. H. \& Lawrence, J. S. (1966) Ann. Rheum. Dis. 26, 497-507.

5. Dezelic, G., Dürrigl, T., Dezelic, N., Zergollern, V., Jurak, H., Vitans, M. \& Androic, S. (1978) Z. Rheumatol. 37, 112-122.

6. Pope, R. M. \& Duffy, S. J. (1979) Arthritis Rheum. 9, 988998.

7. Rose, M. H., Ragan, C., Pearce, E. \& Lipman, M. O. (1948) Proc. Soc. Exp. Biol. Med. 68, 1-6.

8. Waaler, E. (1940) Acta Patol. Microbiol. Scand. 17, 172187.

9. Singer, J. M. \& Plotz, C. M. (1956) Am. J. Med. 21, 888892.

10. Carson, D. A., Lawrence, S., Catalno, M. A., Vaugham, J. H. \& Abraham, G. (1977) J. Immunol. 1, 295-300.

11. Hay, F. C., Nineham, L. J. \& Roitt, M. L. (1975) Br. Med. J. 3, 203-204.

12. Heidelberger, M. (1956) Lectures in Immunochemistry. Acad. Press, New York.
13. Knez, V. \& Reimer, C. H. (1977) J. Immunol. Meth. 18, $105-121$.

14. Nineham, L. J., Hay, F. C. \& Roitt, I. M. (1976) J. Clin. Pathol. 29, 1121-1126.

15. Lea, D. J. \& Ward, D. J. (1978) Ann. Rheum. Dis. 37, 247251.

16. Schulze, A. \& Geiler, G. (1979) Z. Rheumatol. 38, 50-58.

17. Singh, I. \& Francis, G. E. (1978) J. Clin. Pathol. 31, 963973.

18. Grippenberg, M., Wafin, F., Isomaki, H. \& Linder, E. (1979) J. Immunol. Meth. 31, 109-118.

19. Maiolini, R., Ferrura, B., Quaranta, J. F., Pinoteau, A., Euller, L., Ziegler, G. \& Masseyeff, R. (1978) J. Immunol. Meth. 20, 25-34.

20. Finley, R. P., Hicks, M. J., Williams, R. J., Hinlicky, J. \& Lichti, D. (1979) Clin. Chem. 11, 1909-1914.

21. Jones, C. E., Rosseau, R. J. \& Maxwell, K. W. (1979) Am. J. Clin. Pathol: 72, 432-436.

22. Schmolke, B., Leyssens, H. \& Vorlaender, K. O. (19.77) Diagnostik 10, 865-869.

23. Blume, P. \& Greenberg, L. J. (1975) Clin. Chem. 9, $1234-$ 1237.

Dr. med. Klaus Husmann Medizinische Klinik C der Universität Düsseldorf Moorenstr. 5 D-4000 Düsseldorf̣ 